Pacific Journal of Mathematics

TOTALLY BOUNDED GROUP TOPOLOGIES AND CLOSE I 


\title{
TOTALLY BOUNDED GROUP TOPOLOGIES AND CLOSED SUBGROUPS
}

\author{
S. JANAKIRAMAN AND T. SoundaraRAJAN
}

Let $(G, J)$ be an infinite compact totally disconnected abelian group. Finer totally bounded group topologies $J^{\prime}$ such that every $J^{\prime}$-closed subgroup is $J$-closed are studied. Necessary and sufficient conditions for the existence of such a $J^{\prime} \neq J$ are given.

Introduction. Throughout this paper all topologies are Hausdorff topological group topologies and all the groups are written in the additive notation.

A topological group $G$ is called totally bounded if for every identity neighburhood $V$ there is a finite subset $F$ of $G$ with $G=$ $F+V$. This is tantamount to saying that $G$ is embedded algebraically and topologically into its Bohr compactification under the natural map $G \rightarrow \alpha G$. We recall that for abelian $G$ we have $\alpha G=$ $\left(\left(G^{\wedge}\right)_{d}\right)^{\wedge}$ and that $\left(G^{\wedge}\right)_{d}=(\alpha G)^{\wedge}$.

Now let $G$ be a compact abelian group with topology $J$ and let $G^{\prime}$ be the same underlying group with a possibly finer totally bounded topology $J^{\prime}$. Then $G^{\wedge} \subseteq\left(G^{\prime}\right)^{\wedge}=\left(G^{\prime \wedge}\right)_{d} \subseteq\left(G_{d}\right)^{\wedge}$; and conversely, any group $H$ of (not necessarily continuous) characters of $G$ with $G^{\wedge} \subseteq$ $H \subseteq\left(G_{d}\right)^{\wedge}$ induces on $G$ a coarsest topology $J^{\prime}$ making all characters of $H$ continuous, and then the group $G^{\prime}$ with the topology $J^{\prime}$ is totally bounded such that $\left(\alpha G^{\prime}\right)^{\wedge}=G_{d}^{\prime \wedge}=H$. Thus there is a lattice isomorphism between the lattice of totally bounded topologies $J^{\prime}$ on $G$ refining $J$ and the lattice of subgroups of $\left(G_{d}\right)^{\wedge}$ containing $G^{\wedge}$. (These nice results are proved by W. W. Comfort and K. A. Ross in [1].) Furthermore, the diagram

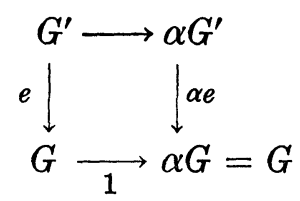

shows that $\alpha G^{\prime}$ is algebraically the direct sum of the image of $G^{\prime}$ in $\alpha G^{\prime}$ and of ker $\alpha e$.

The problem we are interested in studying is the following:

(P) Determine all those totally bounded topologies $J^{\prime}$ containing $J$ such that every $J^{\prime}$-closed subgroup of $G^{\prime}$ is a $J$-closed subgroup of $G$.

In view of the isomorphism of lattices mentioned before, this 
is tantamount to the following problem:

$\left(\mathrm{P}^{\prime}\right)$ Determine all those intermediate groups $H$ with $G^{\wedge} \subseteq H \subseteq$ $\left(G_{d}\right)^{\wedge}$ for which the associated topology $J^{\prime}=J_{H}$ has the same closed subgroups as $J=J_{G}$.

In a totally bounded group the smallest closed subgroup containing a subset $S$ is its bipolar $S^{\perp \perp}$; hence a subgroup $S$ is closed if and only if it agrees with its bipolar if and only if it is the intersection of a collection of kernels of continuous characters. As a consequence, the $J_{H}$-closed subgroups are precisely the intersections of families of groups $\operatorname{ker} f$ with $f \in H$. Consequently problem $\left(\mathrm{P}^{\prime}\right)$ is equivalent to $\left(\mathrm{P}^{\prime \prime}\right)$ : Determine all those groups $H$ with $G^{\wedge} \subseteq$ $H \subseteq\left(G_{d}\right)^{\wedge}$ such that ker $f$ is $J=J_{G}^{\wedge}-$ closed for all $f \in H$.

In this paper we consider only the case $(G, J)$ is totally disconnected ie $G^{\wedge}$ is a torsion group [5, p. 385]. We show that if $H$ is a subgroup "admissible" in the sense of problem $\left(\mathrm{P}^{\prime}\right)$ then $G^{\wedge}$ is the torsion subgroup of $H$ [Lemma 1.3]. In particular ker $\alpha e$ is always connected in this case. Next for any $f \in\left(G_{d}\right)^{\wedge}$ whose $\operatorname{ker} f$ is $J$ closed, $G^{\wedge}+\langle f\rangle$ is admissible [Lemma 2.3]. We then prove that $(G, J)$ has an admissible $H \supseteqq G^{\wedge}$ if and only if $G$ has a direct factor which is $p$-adic integer group $\Delta_{p}$ or an infinite product of cyclic groups of prime power order for infinitely many different primes. [Theorem 2.5].

It is also shown that if there are admissible groups properly containing $G^{\wedge}$ then there is no largest admissible $H$ [Theorem 2.10].

That one can never expect pseudocompact $J^{\prime} \neq J$ (whether or not $J$ is totally disconnected) and existence of maximal admissible subgroups $H$ is dealt with in a paper by W. W. Comfort and the second author [3].

The authors conclude the paper with a few remarks on the nonabelian case and a remark on Galois theory (in $\S 3$ ).

The authors are extremely thankful to Professor W. W. Comfort for all his help and encouragement in the preparation of this paper.

The authors are extremely grateful to the referee for the enormous troubles he has taken and we happily record that the present version of the paper owes a deep debt of gratitude to him.

1. Preliminaries. Throughout this paper $(G, J)$ denotes an infinite compact totally disconnected abelian group, $G^{\wedge}$ is the group of all continuous characters of $(G, J), G_{d}$ is the group $G$ endowed with the discrete topology and $\left(G_{d}\right)^{\wedge}$ is the group of all characters on $G$.

Definition 1.1. A subgroup $H$ of $\left(G_{d}\right)^{\wedge}$ is said to be admissible 
if $H$ contains $G^{\wedge}$ and $\operatorname{ker} f$ is a $J$-closed subgroup of $G$ for all $f \in H$.

Proposition 1.2. If $G$ is of finite exponent then the only admissible subgroup is $G^{\wedge}$.

Proof. Let $f \in\left(G_{d}\right)^{\wedge}$ with $\operatorname{ker} f$ a $J$-closed subgroup of $G$. Since $G$ is of finite exponent say $m, m x=0$ for all $x \in G$ yields $f(G)$ is of finite exponent in $T=\boldsymbol{R} / \boldsymbol{Z}$. Hence $f(G)$ is a finite subgroup of $T$. Thus ker $f$ is of finite index in $G$. Already it is $J$-closed. Hence ker $f$ is $J$-open and so we get $f$ is continuous and hence $f \in G^{\wedge}$. The proposition now easily follows.

Lemma 1.3. Let $H$ be an admissible subgroup. Then $G^{\wedge}$ is the torsion subgroup of $H$.

Proof. It is enough to show that if $f \in H$ is of finite order then $f \in G^{\wedge}$. Let $m f=0$. Then $m f(G)=0$. Hence $f(G)$ is of finite exponent in $T$ and so $f(G)$ is a finite group. Hence ker $f$ is of finite index in $G$. It is $J$-closed implies now that it is $J$-open. Hence we get $f$ is continuous and $f \in G^{\wedge}$.

2. In this section we prove the main theorem.

Lemma 2.1. Let $(A, \tau)$ be an abelian totally bounded topological group and $B$ a closed subgroup of $(A, \tau)$. If $B^{\perp}$ is the set of all continuous homomorphisms of $(A, \tau)$ into $T$ which map $B$ to 0 , then $B=\bigcap_{f \in B^{\perp}} \operatorname{ker} f$.

Proof. Let $\alpha A$ be the compact topological group in which $(A, \tau)$ is densely embedded. Then $\alpha A$ is also abelian. Let $\bar{B}$ be the closure of $B$ in $\alpha A$. We have $\bar{B} \cap A=B$. By Pontrjagin-van Kampen duality theory we have $\bar{B}=\bigcap_{f \in \bar{B}^{\perp}} \operatorname{ker} f$. By taking the restrictions of the $f \in \bar{B}^{\perp}$ to $A$, the lemma follows.

LEMMA 2.2. Let $A$ be an abelian group and $f, g$ be two homomorphisms of $A$ into $T$ such that $g$ is of finite order. Let $n$ be any integer. Then ker $(g+n f)$ contains (ker $f \cap \operatorname{ker} g$ ) as a subgroup of finite index.

Proof. Let $g$ be of order $m$. Then for each $x$ in $A, g(m x)=$ $m g(x)=(m g)(x)=0$. Hence $g(A)$ is of finite exponent and so is a finite subgroup of $T$. Consequently ker $f \cap \operatorname{ker} g$ is of finite index in $\operatorname{ker} f$. Easily ker $f \cap \operatorname{ker} g$ is a subgroup of $\operatorname{ker}(g+n f)$. Let $S=\operatorname{ker}(g+n f)$. Then for every $x \in S$ we have $0=(g+n f)(m x)=$ 
$g(m x)+n f(m x)=m g(x)+n m f(x)=m n f(x)$. Now let $B$ be the finite subgroup of order $m n$ in $T$. Then clearly $S \subset f^{-1}(B)$. Also ker $f$ is of finite index in $f^{-1}(B)$. Already $\operatorname{ker} f \cap \operatorname{ker} g$ is of finite index in $\operatorname{ker} f$. Hence $\operatorname{ker} f \cap \operatorname{ker} g$ is of finite index in $f^{-1}(B)$. Since (ker $f \cap \operatorname{ker} g) \subset S \subset f^{-1}(B)$, the lemma follows.

Lemma 2.3. For any $f \in\left(G_{d}\right)^{\wedge} \backslash G^{\wedge}$ such that $\operatorname{ker} f$ is a J-closed subgroup of $G, G^{\wedge}+\langle f\rangle$ is an admissible subgroup.

Proof. It is enough to show that ker $h$ is a $J$-closed subgroup for all $h \in G^{\wedge}+\langle f\rangle$. Now let $h \in G^{\wedge}+\langle f\rangle$. Then $h=g+n f$ with $g \in G^{\wedge}$ and $n$ an integer. As $G^{\wedge}$ is a torsion abelian group $g$ is of finite order. Hence by Lemma 2.2, $\operatorname{ker} g \cap \operatorname{ker} f$ is of finite index in ker $h$. Now ker $g$ is $J$-closed since $g \in G^{\wedge}$ and $\operatorname{ker} f$ is $J$-closed by hypothesis. Hence ( $\operatorname{ker} f \cap \operatorname{ker} g$ ) is a $J$-closed subgroup and ker $h$ is a finite union of cosets of ( $\operatorname{ker} f \cap \operatorname{ker} g)$. Hence $\operatorname{ker} h$ is $J$-closed.

Proposition 2.4. Let $(G, J)$ be one of the following two groups;

(1) $\Delta_{p}$, the topological group of all the p-adic integers with the usual topology, $p$ a prime.

(2) $\prod_{p_{i} \in I} \boldsymbol{Z}\left(p_{i}^{n_{i}}\right)$, the product of cyclic groups of prime power order $p_{i}^{n_{i}}$, with the product topology, where $I$ is an infinite set of primes. (We shall denote this compact group by $C\left(p_{i}, n_{i}\right)$.) Then there is an admissible subgroup $H \neq G^{\wedge}$.

Proof. (1) Algebraically, $\Delta_{p}$ is a torsion free abelian group of cardinality c. Now $T=\sum \boldsymbol{Z}\left(p^{\infty}\right) \oplus \boldsymbol{R}$ algebraically where the sum is extended over all primes [4, p. 105]. $\boldsymbol{R}$ being a torsion free divisible group of cardinality $c$, we can find an algebraic monomorphism $f: \Delta_{p} \rightarrow T$. Clearly $\operatorname{ker} f=0$ is a $J$-closed subgroup. Also $f \notin \Delta_{p}$ since $m f=0$ will imply $f(m x)=0$ for all $x \in \Delta_{p}$ contradicting that $\operatorname{ker} f=0$. Now Lemma 2.3 completes the proof.

(2) For this case we use a product decomposition. Algebraically $T=\Pi Z\left(p^{\infty}\right)$ (see [4, p. 105]) the product extending over all primes. Again we have an algebraic monomorphism $f: G \rightarrow T$; with ker $f=0$, a $J$-closed subgroup. Since $I$ is infinite $G$ has elements of infinite order. Hence $m f=0$ will yield $f(m x)=0$ and contradict $\operatorname{ker} f=0$. Hence $f \notin G^{\wedge}$. Now Lemma 2.3 completes the proof.

THEOREM 2.5. Let $(G, J)$ be an infinite compact totally disconnected abelian topological group. Then the following statements are equivalent.

(1) There exists a totally bounded group topology $J^{\prime}$ containing 
$J$ properly such that every $J^{\prime}$-closed subgroup is $J$-closed.

(2) $G$ has an infinite monothetic factor group.

(3) $G$ has a direct factor $M$ which is either a p-adic group $\Delta_{p}$ or a group $C\left(p_{i}, n_{i}\right)$.

(4) $G$ has an infinite procyclic direct factor.

Proof. $\quad(1) \Rightarrow(2)$. Suppose there exists a totally bounded group topology $J^{\prime}$ on $G$ containing $J$ properly such that every $J^{\prime}$-closed subgroup is $J$-closed. Let $H=\{f: f$ is a continuous homomorphism of $\left(G, J^{\prime}\right)$ into $\left.T\right\}$. Hence there is an $f \in H \backslash G^{\wedge}$. Clearly then $f \in$ $\left(G_{d}\right)^{\wedge} \backslash G^{\wedge}$. Now $f \in H$ implies that $\operatorname{ker} f$ is $J^{\prime}$-closed and so is $J$-closed by hypothesis. Thus $f$ is a discontinuous character for $(G, J)$ with ker $f$ being $J$-closed. Let $\bar{G}=G / \operatorname{ker} f$ and $\bar{J}$ be the quotient topology on $\bar{G}$ obtained from $J$. We now have a monomorphism $\bar{f}: \bar{G} \rightarrow$ $T$ i.e., $(\bar{G})_{d}$ can be injected into $T$. Hence by $[5$, p. 407] the torsion free rank of $(\bar{G})_{d}$ is at most $c$ and the $p$-rank of the torsion subgroup of $(\bar{G})_{d}$ is at most 1 for all $p$. Also $(\bar{G}, \bar{J})$ is an infinite compact totally disconnected abelian group (since $f \notin G^{\wedge}$, $\operatorname{ker} f$ is not $J$-open and hence $\bar{G}$ cannot be finite). Since $\bar{G}^{\wedge}$ is a torsion abelian group let $\bar{G}^{\wedge}=\sum \bar{G}_{p}^{\wedge}, \bar{G}_{p}^{\wedge}$ being the $p$-primary part of $\bar{G}^{\wedge}$. If for some $p, \bar{G}_{p}^{\wedge}$ contains $\boldsymbol{Z}\left(p^{\infty}\right)$ then we get $(\bar{G}, \bar{J})$ has a factor $\Delta_{p}$ and hence $(G, J)$ has a factor group $\Delta_{p}$ which is an infinite compact monothetic group and we are done. Otherwise $\bar{G}_{p}^{\wedge}$ is a reduced group for each $p$. We claim now $\bar{G}_{p}$ is cyclic of prime power order. Otherwise by $\left[4\right.$, p. 117] we can have $\bar{G}_{p}^{\wedge}=\boldsymbol{Z}\left(p^{r}\right) \oplus \boldsymbol{Z}\left(p^{s}\right) \oplus B$ and consequently by duality $(\bar{G}, \bar{J})$ will have a direct factor $\boldsymbol{Z}\left(p^{r}\right) \oplus$ $Z\left(p^{s}\right)$ contradicting that $p$-rank of $(\bar{G})_{d}$ is at most 1 . Thus each $\bar{G}_{p}^{\wedge}$ is cyclic of prime power order and so $\bar{G}^{\wedge}$ is isomorphic to a subgroup of $T$. (See $[5$, p. 407].) Hence $(\bar{G}, \bar{J})$ is monothetic and (2) follows.

$(2) \Rightarrow(3)$. Let $(\bar{G}, \bar{J})$ be an infinite monothetic factor of $(G, J)$. Then $\bar{G}^{\wedge}$ is a torsion subgroup of $T$ (see $[5$, p. 385]).

We consider now $G^{\wedge}$. If $G^{\wedge}$ contains a $Z\left(p^{\infty}\right)$ for some $p$ then $(G, J)$ will have a direct factor $\Delta_{p}$ and we are done. Otherwise $G^{\wedge}$ is a reduced group. Now $\bar{G}^{\wedge}$ is a subgroup of $G^{\wedge}$. Since $\bar{G}^{\wedge}$ is infinite and a subgroup of $T$ and $\bar{G}^{\wedge}$ also has to be reduced we get $\bar{G}_{p}^{\wedge} \neq 0$ for infinitely many $p$. Hence $G_{p}^{\wedge} \neq 0$ for infinitely many $p$. Now applying [4, p. 117] to each of these $G_{p}^{\wedge}$ we easily get $\sum \boldsymbol{Z}\left(p_{i}^{n_{i}}\right)$ is a direct summand of $G^{\wedge}$. Hence $(G, J)$ has a direct factor $C\left(p_{i}\right.$, $\left.n_{i}\right)$. Hence (3) follows.

( 3$) \Rightarrow(1)$. Case (i): Let $(G, J)=N \oplus M, N$ a $J$-closed subgroup, $M$ is topologically isomorphic to $\Delta_{p}$ and the sum direct. Then by the proof of (1) in 2.4 we get easily a homomorphism $f: G \rightarrow T$ such 
that $\operatorname{ker} f=N$ and $f$ is injective on $M$. Surely order of $f$ is infinite and hence $f \notin G^{\wedge}$. Also ker $f$ is $J$-closed. Hence Lemma 2.3 shows that $G^{\wedge}+\langle f\rangle$ is admissible. Thus a $J^{\prime}$ exists by the equivalence in the introduction.

Case (ii): Let $(G, J)=N \oplus M, N$ a $J$-closed subgroup, $M$ is isomorphic to $C\left(p_{i}, n_{i}\right)$ and the sum direct. Then by the proof (2) in 2.4, we get easily a homomorphism $f: G \rightarrow T$ such that $\operatorname{ker} f=N$ and $f$ is injective on $M$. Since $C\left(p_{i}, n_{i}\right)$ has torsion free elements and $f$ is injective on $M$, order of $f$ has to be infinite and so $f \notin G^{\wedge}$. Also $\operatorname{ker} f=N$ is $J$-closed. Thus Lemma 2.3 yields $G^{\wedge}+\langle f\rangle$ is admissible. Hence $J^{\prime}$-exists by the equivalence in the introduction. Thus (1) follows.

$(3) \Rightarrow(4)$. Easy.

$(4) \Rightarrow(2)$. Let $P$ be an infinite procyclic direct factor of $(G, J)$. Then by duality $P^{\wedge}$ is a torsion group which is a direct limit of finite cyclic groups. By [4, p. 58] $\hat{p}$ is locally cyclic. Hence for each prime $p$ the $p$-rank of $P^{\wedge}$ is atmost one. So each $P_{p}^{\wedge}$ is isomorphic to a $\boldsymbol{Z}\left(p^{s}\right), s=0,1, \cdots, \infty$. This yields that $P^{\wedge}$ is isomorphic to a subgroup of $T$ and hence $P$ is monothetic. Thus (2) holds.

Now Theorem 2.5 follows.

We now proceed to discuss the existence of a largest admissible subgroup.

LEMma 2.6. There exists a largest admissible subgroup $L$ if and only if the set of all $f \in\left(G_{d}\right)^{\wedge}$ such that ker $f$ is J-closed form a group. In this case $L$ consists precisely of these.

Proof. Let $L$ be a largest admissible subgroup. Let $f, g \in\left(G_{d}\right)^{\wedge}$ such that $\operatorname{ker} f$, ker $g$ are $J$-closed. Then by Lemma $2.3 G^{\wedge}+\langle f\rangle$ and $G^{\wedge}+\langle g\rangle$ are admissible subgroups; they will both be subgroups of $L$ and hence, $f, g, f-g \in L$. Clearly then all such $f$ 's will form a group.

Conversely let $L=\left\{f \in\left(G_{d}\right)^{\wedge} \mid \operatorname{ker} f\right.$ is $J$-closed $\}$ form a group. Then clearly $L$ is admissible, and by definition any other admissible group should be a subgroup of $L$. Hence the lemma is proved.

Proposition 2.7. In $\left(\left(\Delta_{p}\right)_{d}\right)^{\wedge}$ there is no largest admissible subgroup.

Proof. Since $A_{p}$ is a torsion free abelian group of cardinal $c$ it has a maximal independent set $B$ of cardinal $c$. Hence $\Delta_{p} /\langle B\rangle$ is a torsion abelian group. 
Now $T=\Sigma \boldsymbol{Z}\left(p^{\infty}\right) \oplus \boldsymbol{R}$ (see $[4$, p. 105]). We can write $\boldsymbol{R}=\Sigma Q$, $c$ copies and then write $R=B_{1}+B_{2}$ such that $B_{1} \cap B_{2}=Q, B_{1}, B_{2}$ each isomorphic to $\Sigma Q$, c copies. Now easily we can get embeddings $h_{1}, h_{2}$ of $\Delta_{p}$ into $R$, such that $h_{1}\left(\Delta_{p}\right) \subset B_{1}, h_{2}\left(\Delta_{p}\right) \subset B_{2}$ and $h_{1}(1)=$ $h_{2}(1)=1 \in Q=B_{1} \cap B_{2}, h_{1}, h_{2}$ being obtained by mapping $B$ to the corresponding independent sets. It is easy to see that $\operatorname{ker}\left(h_{1}-h_{2}\right)$ is a countable subgroup, of $\Delta_{p}(=\{n / m ;(p, m)=1\})$. Clearly $\operatorname{ker}\left(h_{1}-h_{2}\right)$ is not $J$-closed. Now Lemma 2.6 completes the proof.

Proposition 2.8. There is no largest admissible subgroup in $\left(C\left(p_{i}, n_{i}\right)_{d}\right)^{\wedge}$.

Proof. We note $C\left(p_{i}, n_{i}\right)=\Pi \boldsymbol{Z}\left(p_{p_{i}}^{n_{i}}\right)$ algebraically and also that $T=\Pi Z\left(p^{\infty}\right), p$ varies over all primes [4, p. 105]. Hence there is an embedding $i: C\left(p_{i}, n_{i}\right) \rightarrow T$, with $\operatorname{ker}(i)=0$; which is $J$-closed. Since $T \cong \sum Z\left(p^{\infty}\right) \oplus R$, there is an automorphism $g: T \rightarrow T$ such that $g(x)=x$ for elements of finite order and $g(x)=\sqrt{2} x$ for $x$ in $\boldsymbol{R}$. Then $g \circ i$ gives another embedding of $C\left(p_{i}, n_{i}\right)$. Now $\operatorname{ker}(i-g \circ i)$ is a countable subgroup namely $\sum \boldsymbol{Z}\left(p_{i}^{n_{i}}\right)$. Thus we get two embeddings $f_{1}, g_{1}: C\left(p_{i}, n_{i}\right) \rightarrow T$ such that $\operatorname{Ker}\left(f_{1}-g_{1}\right)$ is countable and hence not $J$-closed. So Lemma 2.6 completes the proof.

Definition 2.9. We say a topology $J^{\prime}$ is admissible if it satisfies the condition of $(P)$.

THEOREM 2.10. The following are equivalent:

(1) $G$ has a largest admissible topology $J_{L}$,

(2) $G$ has no admissible topology $J^{\prime} \neq J$,

(3) $J$ is the largest admissible topology.

Proof. $(1) \Rightarrow(2)$. Suppose $G$ has an admissible topology $J^{\prime} \neq J$. Then $G$ has a topological decomposition $G=A \oplus B, A$ a closed subgroup and $B$ is isomorphic $\Delta_{p}$ or $C\left(p_{i}, n_{i}\right)$. Then by Propositions 2.7 and 2.8, we have two embeddings of $f, g: B \rightarrow T$ such that $\operatorname{ker}(f-g)$ is countable and not $J$-closed. Hence we easily get two homomorphisms $F_{1}, G_{1}: G \rightarrow T$ such that $\operatorname{ker} F_{1}=\operatorname{ker} G_{1}=A$ is $J$-closed but $\operatorname{ker}\left(F_{1}-G_{1}\right)$ is not $J$-closed. This contradicts Lemma 2.6. Hence (2) follows. $\quad(2) \Rightarrow(3)$ is easy as also $(3) \Rightarrow(1)$.

Proposition 2.11. On $\Delta_{p}$, there is an admissible topology $J^{\prime}$ having $\left|\left(\Delta_{p}, J^{\prime}\right)^{\wedge}\right|=c$.

Proof. We note $T \cong \sum Z\left(p^{\infty}\right) \oplus R$ and $R=\sum Q, c$ copies. Now we can write $R=\sum B_{\alpha}, \alpha \in I ;|I|=c$ and each $B_{\alpha}$ is a torsion free 
divisible abelian group of cardinality $c$. This is possible as $c . c=c$. For each $\alpha \in I$, we can have an embedding $h_{\alpha}=\Delta_{p} \rightarrow B_{\alpha}$. Correspondingly we get embeddings $g_{\alpha}: \Delta_{p} \rightarrow T$ such that for each $x \neq 0$, the $g_{\alpha}(x)$ are independent. Let now $H$ be the subgroup of $\left(\left(\Delta_{p}\right)_{d}\right)^{\wedge}$ generated by $\Delta_{p}^{\hat{p}}$ and all these $g_{\alpha}$. Surely $|H|=c$. Let $J^{\prime}$ be the totally bounded group topology determined by $H . \quad J^{\prime}$ is finer than $J$, the usual topology. We claim $J^{\prime}$ is admissible. We have only to show that $\operatorname{ker}(h)$ is $J$-closed for each $h \in H$, since $H=\left(\Delta_{p}, J^{\prime}\right)^{\wedge}$, (see [5]). Now $h=f+\sum_{1}^{k} n_{i} g_{\alpha_{i}}, f \in \Delta_{p}, n_{i}$ are integers $k$ finite. If all the $n_{i}$ are 0 , then there is nothing to prove. Let some $n_{i} \neq 0$. Since $f$ is of finite order by Lemma 2.2, we have only to prove $\operatorname{ker} f \cap \operatorname{ker}\left(\sum_{1}^{k} n_{i} g_{\alpha_{i}}\right)$ is $J$-closed. We claim $\operatorname{ker}\left(\sum_{1}^{k} n_{i} g_{\alpha_{i}}\right)=0$. Let if possible $x \neq 0$ be in the kernel. $\sum n_{i} g_{\alpha_{i}}(x)=0$ implies $\sum g_{\alpha_{i}}\left(n_{i} x\right)=$ 0 and by independence $g_{\alpha_{i}}\left(n_{i} x\right)=0$ for each $i$ and each $g_{\alpha_{i}}$ being an embedding we get $n_{i} x=0$ for each $i$, so $x=0$. Thus ker $f \cap \operatorname{ker}$ $\left(\sum n_{i} g_{\alpha_{i}}\right)=0, a J$-closed subgroup. Hence the results follows.

3. We now assume $(G, \tau)$ is a noncommutative compact totally disconnected group and make a few remarks on totally bounded group topologies $\tau^{\prime}$ containing $\tau$ and such that each $\tau^{\prime}$ closed subgroup is $\tau$-closed. We shall again call such a $\tau^{\prime}$ an admissible topo$\log y$.

REMARK 3.1. If $G$ is of finite exponent then $\tau^{\prime}=\tau$.

Proof. Let $\alpha G^{\prime}$ be a compact topological group in which $\left(G, \tau^{\prime}\right)$ is embedded as a dense subgroup. From hypothesis it now follows easily that for each $x \in \alpha G^{\prime}, m x=0$ (since $x$ is limit of a net, from $\left.\left(G, \tau^{\prime}\right)\right)$. Now $\bigcap_{n=1}^{\infty} n\left(\alpha G^{\prime}\right)=0$, since $m \alpha G^{\prime}=0$. Hence by a theorem of Mycielski [8], $\alpha G^{\prime}$ is totally disconnected and hence by [7, p. 56] has a basis of open subgroups of finite index at 0 .

Hence $\left(G, \tau^{\prime}\right)$ has a basis $\left\{G_{\alpha}^{\prime}\right\}$ of open subgroups of finite index at 0 . Each of these $G_{\alpha}^{\prime}$ is now $\tau$-closed and hence $\tau$-open. Hence we get $\tau$ is finer than $\tau^{\prime}$. Since $\tau$ is compact and $\tau^{\prime}$ is Hausdorff we get $\tau=\tau^{\prime}$.

REMARK 3.2. Let $(K, \tau)$ be a compact group of finite exponent. Then $K \times \Delta_{p}$ has an admissible topology different from the product topology.

Proof. Let $m x=0$ for each $x \in K$. Let $J_{1}$ be an admissible topology on $\Delta_{p} ; J_{1} \neq$ the usual topology $J$ of $\Delta_{p}$. Let $J^{\prime}$ be the product of $\tau$ and $J_{1}$ on $K \times \Delta_{p}$. Since $\left(K \times \Delta_{p}, J^{\prime}\right) \subset(K, \tau) \times\left(\alpha \Delta_{p}, \alpha J_{1}\right)$ where $\alpha \Delta_{p}$ is the compact group in which $\Delta_{p}$ is densely embedded, 
we get $J^{\prime}$ is totally bounded. Also $J^{\prime}$ is finer than the product topology $\tau \times J$. We have only to show that any $J^{\prime}$-closed subgroup $S$ is $\tau \times J$ closed. If $S \subset(K \times O)$ then we easily get the result. Suppose $S \not \subset K \times 0$. If $(x, y) \in S \backslash(K \times 0)$ then $m(x, y)=(m x, m y)=$ $(0, m y) \in 0 \times \Delta_{p}$. Let $S \cap\left(0 \times \Delta_{p}\right)=M \neq(0,0)$ and $S \cap(K \times 0)=M_{1}$. $M$. is a $J^{\prime}$-closed subgroup of $\Delta_{p}$ and hence $J$-closed. So $M=0 \times$ $p^{n} \Delta_{p}$ for some $n . \quad M_{1}$ is $J^{\prime}$-closed and hence $J$-closed since $K \times 0$ is $J^{\prime}$-closed and $J^{\prime}=\tau \times J$ on $K \times 0$. Now $M_{1} \times M$. is $J$-closed and $\subset S$. We claim $M_{1} \times M$ is of finite index in $S$. Let $p_{1}: S \rightarrow \Delta_{p}$ be the projection. Then $p_{1}(S) \supset M$. Hence $M$ is of finite index in $p_{1}(S)$ (since $M$ is of finite index $p^{n}$ in $\Delta_{p}$ itself). Let $p_{1}(S)=M \cup$ $\left(a_{2}+M\right) \cup \cdots \cup\left(a_{k}+M\right)$ where $\left(y_{i}, a_{i}\right) \in S, i=1,2, \cdots, k$. We claim now $S=U_{1}^{k}\left(\left(y_{i}, a_{i}\right)+\left(M_{1} \times M\right)\right)$. Let $(x, y) \in S$. Then $p_{1}(x, y)=y=$ $a_{i}+t$ for some $i$ and $t \in M$. Also $(0, t) \in M_{1} \times M \subset S$. Hence we can assume $p_{1}(x, y)=a_{i}$. Also $p_{1}\left(y_{i}, a_{i}\right)=a_{i}$. Hence $\left(-\left(y_{i}, a_{i}\right)+(x, y)\right)=$ $\left(-y_{i}+x, 0\right) \in M_{1} \subset M_{1} \times M$. Hence $(x, y) \in\left(y_{i}, a_{i}\right)+\left(M_{1} \times M\right)$. Hence $S$ is a finite union of cosets of $M_{1} \times M$ and so we get $S$ is $\tau \times J$ closed. That $\tau \times J_{1}$ is an admissible topology follows now easily.

REMARK 3.3. If $E$ is an infinite algebraic separable normal extension of a field $F$ and $G$ is the Galois group of $E$ over $F$ then W. Krull [6] has shown that one can introduce a topology $\tau$ on $G$ (the Krull topology) such that there is a $1-1$ Galois correspondence between all intermediate fields of $E$ over $F$ and all $\tau$-closed subgroups of $G$. Furthermore $(G, \tau)$ is a compact totally disconnected group. It might be of some interest that if $\tau^{\prime}$ is any other admissible topology on $G$ then again there is a $1-1$ Galois correspondence between all intermediate fields of $E$ over $F$ and all $\tau^{\prime}$-closed subgroups of $G$.

\section{REFERENCES}

1. W. W. Comfort and K. A. Ross, Topologies induced by groups of characters, Fund. Math., 55 (1964), 283-291.

2. W. W. Comfort and V. Saks, Countably compact groups and finest totally bounded topologies, Pacific J. Math., 49 (1973), 33-44.

3. W. W. Comfort and T. Soundararajan, Pseudocompact group topologies and totally dense subgroups (submitted to the Pacific Journal of Mathematics).

4. L. Fuchs, Infinite Abelian Groups, I, Academic Press, New York, 1970.

5. E. Hewitt and K. A. Ross, Abstract Harmonic Analysis, I, Springer-Verlag, New York, 1963.

6. W. Krull, Galoissche Theorie der unendlichen algebraischen Erweiterungen, Math, Ann., 100 (1928), 687-698.

7. D. Montgomery and L. Zippin, Topological transformation groups, Interscience, 1955.

8. J. Mycielski, Some properties of connected compact groups, Colloq. Math., 5 (1957), 162-166.

9. K. A. Ross, Closed subgroups of locally compact Abelian groups, Fund. Math., 56 
(1965), 241-244.

10. A. Weil, L' intégration dans les groups topologiques et ses applications, Hermann, Paris, 1951.

Received February 18, 1981 and in revised form June 30, 1981.

Madurai Kamaraj University

MAdURAI 625021 INDIA 


\section{PACIFIC JOURNAL OF MATHEMATICS}

\section{EDITORS}

DONALD BABBITT (Managing Editor)

J. DUGUNDJI

University of California

Los Angeles, California 90024

Hugo Rossi

University of Utah

Salt Lake City, UT 84112

C. C. Moore and Arthur Agus

Department of Mathematics

University of Southern California

Los Angeles, California 90007

R. FinN and J. Milgram

Stanford University

Stanford, California 94305

University of California

Berkeley, CA 94720

\section{ASSOCIATE EDITORS}
R. ARNES
E. F. BeCKenBaCH
B. H. NEUMANN
F. WoLF
K. YoshidA

\section{SUPPORTING INSTITUTIONS}

UNIVERSITY OF ARIZONA

UNIVERSITY OF BRITISH COLUMBIA

CALIFORNIA INSTITUTE OF TECHNOLOGY

UNIVERSITY OF CALIFORNIA

MONTANA STATE UNIVERSITY

UNIVERSITY OF NEVADA, RENO

NEW MEXICO STATE UNIVERSITY

OREGON STATE UNIVERSITY
UNIVERSITY OF OREGON

UNIVERSITY OF SOUTHERN CALIFORNIA

STANFORD UNIVERSITY

UNIVERSITY OF HAWAII

UNIVERSITY OF TOKYO

UNIVERSITY OF UTAH

WASHINGTON STATE UNIVERSITY

UNIVERSITY OF WASHINGTON 


\section{Pacific Journal of Mathematics}

\section{Vol. 101, No. $1 \quad$ November, 1982}

Natália Bebiano, On the evaluation of permanents $\ldots \ldots \ldots \ldots \ldots \ldots \ldots \ldots$

David Borwein and Bruce Brigham Watson, Tauberian theorems between

the logarithmic and Abel-type summability methods $\ldots \ldots \ldots \ldots \ldots \ldots 11$

Leo George Chouinard, II, Hermite semigroup rings $\ldots \ldots \ldots \ldots \ldots \ldots \ldots$

Kun-Jen Chung, Remarks on nonlinear contractions $\ldots \ldots \ldots \ldots \ldots \ldots . \ldots 4$

Lawrence Jay Corwin, Representations of division algebras over local

fields. II ......................................... 49

Mahlon M. Day, Left thick to left lumpy—a guided tour $\ldots \ldots \ldots \ldots \ldots 71$

M. Edelstein and Mo Tak Kiang, On ultimately nonexpansive

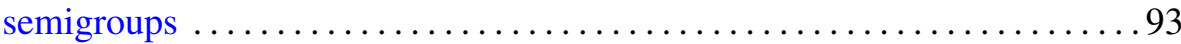

Mary Rodriguez Embry, Semigroups of quasinormal operators . ........ 103

William Goldman and Morris William Hirsch, Polynomial forms on

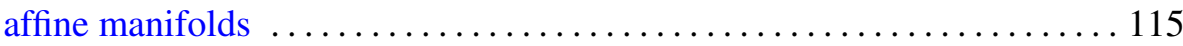

S. Janakiraman and T. Soundararajan, Totally bounded group topologies

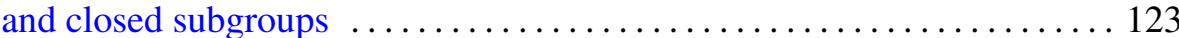

John Rowlay Martin, Lex Gerard Oversteegen and Edward D.

Tymchatyn, Fixed point set of products and cones $\ldots \ldots \ldots \ldots \ldots \ldots 133$

Jan van Mill, A homogeneous Eberlein compact space which is not metrizable ........................................ 141

Steven Paul Plotnick, Embedding homology 3-spheres in $S^{5} \ldots \ldots \ldots \ldots 147$

Norbert Riedel, Classification of the $C^{*}$-algebras associated with minimal rotations

Benedict Seifert, Combinatorial and geometric properties of weight systems of irreducible finite-dimensional representations of simple split Lie algebras over fields of 0 characteristic

James E. Simpson, Dilations on locally convex spaces

Paolo M. Soardi, Schauder bases and fixed points of nonexpansive mappings

Yoshio Tanaka, Point-countable $k$-systems and products of $k$-spaces

Fausto A. Toranzos, The points of local nonconvexity of starshaped sets . . . 209

Lorenzo Traldi, The determinantal ideals of link modules. I . . . . . . . . 215

P. C. Trombi, Invariant harmonic analysis on split rank one groups with applications

Shinji Yamashita, Nonnormal Blaschke quotients 